\title{
NONEXISTENCE OF MEASURABLE OPTIMAL SELECTIONS
}

\author{
JOHN BURGESS AND ASHOK MAITRA
}

(Communicated by Lawrence F. Gray)

\begin{abstract}
We give an example of a function $f$ on a separable metric space $X$ into a compact metric space $Y$ such that the graph of $f$ is a Borel subset of $X \times Y$, but $f$ is not Borel measurable. The example forms the basis for our construction of an upper semicontinuous, compact model of a one-day dynamic programming problem where the player has an optimal action at each state, but is unable to make a choice of such an action in a Borel measurable manner.
\end{abstract}

\section{INTRODUCTION}

A very useful result for dynamic programming/gambling/discrete time stochastic control is the following selection theorem due to Schäl $[8,9]$ :

Theorem A. Suppose $(T, \mathscr{T})$ is a measurable space, and let $Y$ be a separable metric space. Suppose $G$ is a $\mathscr{T}$-measurable multifunction on $T$ such that $G(t)$ is a nonempty, compact subset of $Y$ for every $t \in T$. Let $v$ be a real-valued function on $\operatorname{Graph}(G)(=\{(t, y) \in T \times Y: y \in G(t)\})$ satisfying the following condition:

(1) $v$ is the pointwise limit of a nonincreasing sequence $\left\{v_{n}\right\}$ of real-valued functions on $\operatorname{Graph}(G)$ such that each $v_{n}$ is $\left(\mathscr{T} \times \mathbf{B}_{y} \mid \operatorname{Graph}(G)\right)$-measurable and $v_{n}(t, \cdot)$ is continuous on $G(t)$ for every $t \in T$, where $\mathbf{B}_{y}$ is the Borel $\sigma$-field on $Y$. Let

$$
v^{*}(t)=\sup _{y \in G(t)} v(t, y), \quad t \in T .
$$

Then there is a $\mathscr{T}$-measurable selector $g: T \rightarrow Y$ of $G$ such that $v^{*}(t)=$ $v(t, g(t))$ for every $t \in T$.

We recall that to say $G$ is $\mathscr{T}$-measurable means that for every open subset $V$ of $Y$, the set $\{t \in T: G(t) \cap V \neq \varnothing\}$ belongs to $\mathscr{T}$. Since $G$ is compact valued, this condition implies the apparently stronger condition that for every closed subset $C$ of $Y$, the set $\{t \in T: G(t) \cap C \neq \varnothing\}$ belongs to $\mathscr{T}$.

In the dynamic programming literature, theorems of the above type are called Dubins-Savage selection theorems. Theorem A is the culmination of many attempts (see, for instance $[4,11]$ ) to improve on the original result of Dubins and Savage [1]. Many infinite horizon problems of dynamic programming can

Received by the editors April 15, 1991.

1991 Mathematics Subject Classification. Primary 90C39, 28B20; Secondary 04A15.

Key words and phrases. Measurable selections, Borel sets and functions, dynamic programming. 
be reduced to a one-day problem by use of the Optimality Equation. Once this is done, the situation becomes like that described in Theorem A. One can think of $T$ as the state space, $G(t)$ as the set of actions available to the player when the system is in the state $t$, and $v(t, y)$ as the reward when the player chooses action $y$ in state $t$. Theorem A then states that the player can choose an optimal action in each state in a measurable manner. This optimal rule can then be used every day to define an optimal stationary policy in the infinite horizon problem (see, e.g., $[4,9]$ for details).

It is not unreasonable to conjecture that Theorem A remains true if condition (1) is replaced by the weaker condition

$\left(1^{\prime}\right) v$ is $\left(\mathscr{T} \times \mathbf{B}_{y} \mid \operatorname{Graph}(G)\right)$-measurable and $v(t, \cdot)$ is upper semicontinuous (u.s.c.) on $G(t)$ for every $t \in T$.

The aim of this article is to give a counterexample to this conjecture, even when $T$ is a separable metric space and $\mathscr{T}$ is its Borel $\sigma$-field.

\section{CODING OF BOREL SETS}

Our basic example will be based on a coding of Borel subsets of $\mathbf{R}$, the real line, due to Solovay [10, pp. 25-28]. The coding will now be described in some detail. The main properties of the coding will be stated in a sequence of lemmas. The proofs of the lemmas can be found in [10].

In what follows, $\omega$ will denote the set of natural numbers. Let $\left\{r_{i}\right\}$ be an enumeration of the rationals and let $J$ be the pairing function on $\omega \times \omega$ defined by $J(a, b)=2^{a}(2 b+1)$. We define the coding recursively as follows:

1. $\alpha \in \omega^{\omega}$ codes $\left[r_{i}, r_{j}\right]$ if $\alpha(0) \equiv 0(\bmod 3), \alpha(1)=i$, and $\alpha(2)=j$.

2. Suppose $\alpha_{i} \in \omega^{\omega}$ codes $B_{i} \subseteq \mathbf{R}, i=0,1, \ldots$; then $\alpha \in \omega^{\omega}$ codes $U_{i} B_{i}$ if $\alpha(0) \equiv 1(\bmod 3)$ and $\alpha(J(a, b))=\alpha_{a}(b)$.

3. Suppose $\beta \in \omega^{\omega}$ codes $B, \alpha(0)=2(\bmod 3)$, and $\alpha(n+1)=\beta(n)$. Then $\alpha$ codes $B^{c}$, the complement of $B$.

4. $\alpha \in \omega^{\omega}$ codes $B \subseteq \mathbf{R}$ only as required by $1-3$.

Lemma 1. (a) Every $\alpha \in \omega^{\omega}$ codes at most one subset of $\mathbf{R}$.

(b) Every Borel subset of $\mathbf{R}$ is coded by some $\alpha \in \omega^{\omega}$.

(c) If a subset of $\mathbf{R}$ is coded by $\alpha$, then it is Borel.

Next, we define a function $\Phi: \omega^{\omega} \times \omega \rightarrow \omega^{\omega}$, with the property that if $\alpha$ codes a Borel set $B$, then $\Phi(\alpha, \cdot)$ recovers the Borel sets from which $B$ is constructed. For the definition, we need to fix an enumeration $\left\{s_{n}\right\}$ without repetitions, of finite sequences of natural numbers (including the empty sequence) such that if $s_{n}$ is an initial segment of $s_{m}$, then $n \leq m$ So $s_{0}$ is the empty sequence. The definition of $\Phi(\alpha, n)$ will proceed by induction on $n$.

Set $\Phi(\alpha, 0)=\alpha, \alpha \in \omega^{\omega}$. Let $n>0$ and suppose that $\Phi(\alpha, m)$ has been defined for all $\alpha \in \omega^{\omega}$ and all $m<n$. Since $n>0, s_{n}$ is a finite sequence of positive length $k$, say. Let $s_{m}$ be the initial segment of $s_{n}$ of length $(k-1)$, so $m<n$; let $u$ be the last coordinate of $s_{n}$. Now define for $i \in \omega$

$$
\Phi(\alpha, n)(i)= \begin{cases}0 & \text { if } \Phi(\alpha, m)(0) \equiv 0(\bmod 3), \\ \Phi(\alpha, m)(J(u, i)) & \text { if } \Phi(\alpha, m)(0) \equiv 1(\bmod 3), \\ \Phi(\alpha, m)(i+1) & \text { if } \Phi(\alpha, m)(0) \equiv 2(\bmod 3) .\end{cases}
$$


Lemma 2. (a) $\Phi$ is Borel measurable.

(b) If $\alpha$ codes a Borel set, then for all $n, \Phi(\alpha, n)$ codes a Borel set.

For $\beta \in \omega^{\omega}$, define $\bar{\beta} \in \omega^{\omega}$ so that for every $n \in \omega$,

$$
s_{\bar{\beta}(n)}=(\beta(0), \beta(1), \ldots, \beta(n-1)) .
$$

Plainly, the map $\beta \rightarrow \bar{\beta}$ is continuous.

Let $C=\left\{\alpha \in \omega^{\omega}:(\forall \beta)(\exists n) \Phi(\alpha, \bar{\beta}(n)) \equiv 0\right\}$.

Lemma 3. (a) $C$ is a coanalytic subset of $\omega^{\omega}$.

(b) $C$ is the set of all $\alpha \in \omega^{\omega}$ such that $\alpha$ codes a Borel subset of $\mathbf{R}$. by $i$.

Define a function $\varphi: \omega \times \omega \rightarrow \omega$ such that $s_{\varphi(n, i)}$ is the sequence $s_{n}$ followed

Let $E \subseteq \omega^{\omega} \times \mathbf{R} \times 2^{\omega}$ be defined as follows:

$$
\begin{aligned}
& (\alpha, x, \gamma) \in E \leftrightarrow(\forall n)[\{\Phi(\alpha, n)(0) \equiv 0(\bmod 3) \\
& \quad \rightarrow\left\{\gamma(n)=1 \leftrightarrow(\exists i)(\exists j)\left(\Phi(\alpha, n)(1)=i \& \Phi(\alpha, n)(2)=j \& x \in\left[r_{i}, r_{j}\right]\right\}\right] \\
& \quad \&(\forall n)[\{\Phi(\alpha, n)(0) \equiv 1(\bmod 3) \rightarrow\{\gamma(n)=1 \leftrightarrow(\exists i)(\gamma(\varphi(n, i))=1)\}] \\
& \quad(\forall n)[\{\Phi(\alpha, n)(0) \equiv 2(\bmod 3) \rightarrow\{\gamma(n)=1 \leftrightarrow \gamma(\varphi(n, 0))=0\}]
\end{aligned}
$$

Lemma 4. (a) $E$ is a Borel subset of $\omega^{\omega} \times \mathbf{R} \times 2^{\omega}$.

(b) For every $\alpha \in C$ and $x \in \mathbf{R}$, there is a unique $\gamma \in 2^{\omega}$ such that $(\alpha, x, \gamma) \in E$.

(c) If $\alpha \in C$ and $(\alpha, x, \gamma) \in E$, then $(\forall n)(\gamma(n)=1 \leftrightarrow x \varepsilon$ the Borel set coded by $\Phi(\alpha, n))$.

This completes our description of Solovay's coding of Borel subsets of $\mathbf{R}$.

\section{CountereXamples}

We use the notation introduced in the previous section.

Let $f$ be the function on $C \times \mathbf{R}$ into $2^{\omega}$ guaranteed by Lemma $4(\mathrm{~b})$; that is, for $\alpha \in C$ and $x \in \mathbf{R}, f(\alpha, x)$ is the unique $\gamma$ such that $(\alpha, x, \gamma) \in E$. It follows that

$$
\operatorname{Graph}(f)=E \cap\left(C \times \mathbf{R} \times 2^{\omega}\right)
$$

so that $\operatorname{Graph}(f)$ is (relatively) Borel in $C \times \mathbf{R} \times 2^{\omega}$. We will now show that $f$ is not Borel measurable. Towards a contradiction, assume that $f$ is Borel measurable on $C \times \mathbf{R}$. Consider the set

$$
F=\{(\alpha, x) \in C \times \mathbf{R}: f(\alpha, x)(0)=0\} .
$$

According to Lemmas $3(\mathrm{~b})$ and $4(\mathrm{c})$ and the definition of $\Phi$, the condition " $\alpha \in C \& f(\alpha, x)(0)=0$ " states that $x$ does not belong to the Borel set coded by $\alpha$. Since $f$ is Borel measurable, $F$ is (relatively) Borel in $C \times \mathbf{R}$ so there must exist a Borel subset $D$ of $\omega^{\omega} \times \mathbf{R}$ such that $F=D \cap(C \times \mathbf{R})$. Fix a homeomorphism $h$ from the set $I$ of irrationals in $\mathbf{R}$ onto $\omega^{\omega}$. Let $B=\{x \in I:(h(x), x) \in D\}$. Plainly, $B$ is a Borel subset of $\mathbf{R}$. So, by Lemmas $1(\mathrm{~b})$ and 3(b), there is $\alpha^{*} \in C$ such that $\alpha^{*}$ codes $B$. Set $x^{*}=h^{-1}\left(\alpha^{*}\right)$. Then

$$
\begin{aligned}
x^{*} \in B & \leftrightarrow\left(\alpha^{*}, x^{*}\right) \in D \leftrightarrow\left(\alpha^{*}, x^{*}\right) \in F \leftrightarrow f\left(\alpha^{*}, x^{*}\right)(0)=0 \\
& \leftrightarrow x^{*} \notin \text { the Borel set coded by } \alpha^{*} \leftrightarrow x^{*} \notin B,
\end{aligned}
$$

a contradiction! 
Since the domain of the function $f$ is coanalytic (Lemma $3(a)$ ) and $2^{\omega}$ is homeomorphic to the Cantor set, the above construction shows that the Measurable Graph Theorem fails, in general, for a function with coanalytic domain $X$ and values in $[0,1]$. That is, such a function can have a graph that is a Borel subset of $X \times[0,1]$, but it is not necessarily the case that the function is Borel measurable. A fortiori, a multifunction with coanalytic domain and nonempty, compact subsets of $[0,1]$ as values can have a Borel measurable graph without being Borel measurable. This answers a question of some interest to workers in stochastic optimization (see, e.g., [6]). On the other hand, it is a classical result (see [3]) that Borel measurability of the graph of a function with analytic domain and values in a Polish space implies the Borel measurability of the function. This is also true, courtesy of the Kunugui-Novikov theorem [3], of a multifunction with analytic domain and nonempty, compact values in a Polish space.

It is now easy to turn the basic example into the desired counterexample to Theorem A with condition (1) replaced by $\left(1^{\prime}\right)$.

Let $T=C \times \mathbf{R}, \mathscr{T}=$ the Borel $\sigma$-field of $C \times \mathbf{R}, Y=2^{\omega}, G(t)=2^{\omega}$ for every $t \in T$, and

$$
v((\alpha, x), \gamma)= \begin{cases}1 & \text { if } f(\alpha, x)=\gamma, \\ 0 & \text { otherwise. }\end{cases}
$$

Then $v$ is Borel measurable on $\operatorname{Graph}(G)=T \times Y$, since $\operatorname{Graph}(f)$ is a Borel subset of $T \times Y$. Moreover for fixed $t \in T, v(t, \cdot)$ is u.s.c. on $G(t)$. Thus, condition $\left(1^{\prime}\right)$ is satisfied. But, plainly, there is no Borel measurable selector $g$ of $G$ such that $v^{*}(t)=v(t, g(t))$ for all $t \in T$.

In the example above, the function $v^{*}$, being identically equal to one, is $\mathscr{T}$-measurable. We now modify the example so that $v^{*}$ is not $\mathscr{T}$-measurable.

Fix a Borel isomorphism of the Borel set $E$ and the interval $[1 / 2,1]$ and denote by $\psi$ the restriction of the Borel isomorphism to $\operatorname{Graph}(f)$, so that $\psi$ continues to be a Borel isomorphism of $\operatorname{Graph}(f)$ and the range of $\psi$. As before, take $T=C \times \mathbf{R}, \mathscr{T}=$ the Borel $\sigma$-field of $C \times \mathbf{R}, Y=2^{\omega}, G(t)=2^{\omega}$ for every $t \in T$, and

$$
v((\alpha, x), \gamma)= \begin{cases}\psi(\alpha, x, \gamma) & \text { if } f(\alpha, x)=\gamma, \\ 0 & \text { otherwise }\end{cases}
$$

It is easily checked that condition $\left(1^{\prime}\right)$ is satisfied. Next observe that

$$
v^{*}((\alpha, x))=\psi(\alpha, x, f(\alpha, x))
$$

for all $(\alpha, x) \in T$. If $v^{*}$ were $\mathscr{T}$-measurable, then the map $(\alpha, x) \rightarrow$ $(\alpha, x, f(\alpha, x))$ would be $\mathscr{T}$-measurable, since $\psi$ is a Borel isomorphism. But then $f$ would be $\mathscr{T}$-measurable, a contradiction!

We remark that if $T$ is an analytic set and $\mathscr{T}$ the Borel $\sigma$-field of $T$, then condition $\left(1^{\prime}\right)$ implies condition (1) and so Theorem $\mathrm{A}$ is true under condition $\left(1^{\prime}\right)$. To see that $\left(1^{\prime}\right)$ implies (1), fix a metric $\rho$ on $Y$ and define for $n=1,2, \ldots$,

$$
v_{n}(t, y)=\sup _{y^{\prime} \in G(t)}\left\{v\left(t, y^{\prime}\right)-n \rho\left(y^{\prime}, y\right)\right\}, \quad(t, y) \in \operatorname{Graph}(G) .
$$

The only nontrivial thing to check regarding condition (1) is that $v_{n}$ is $(\mathscr{T} \times$ $B_{Y} \mid \operatorname{Graph}(G)$ )-measurable and this follows from the Kunugui-Novikov theorem 
[3]. The validity of Theorem A under condition $\left(1^{\prime}\right)$ when $T$ is analytic and $\mathscr{T}$ is its Borel $\sigma$-field was observed by Himmelberg et al. [2] and also by Schäl [11].

\section{Proof of Theorem A}

For the sake of completeness, we give a quick proof of Theorem A. Our proof differs from those of Schäl [8,9] and Reider [7]. We start with an easy lemma.

Lemma B. Let $Z$ be a compact metric space. Suppose $f, f_{m}: Z \rightarrow \mathbf{R}$ are u.s.c. and $f_{m} \downarrow f$. If $z_{m} \rightarrow z_{0}$ in $Z$, then $\lim \sup _{m} f_{m}\left(z_{m}\right) \leq f\left(z_{0}\right)$.

Proof. Let $\varepsilon>0$. Choose a continuous function $g: Z \rightarrow \mathbf{R}$ such that $g \geq f$ and $f\left(z_{0}\right) \geq g\left(z_{0}\right)-\varepsilon$.

Set $g_{m}=\max \left(f_{m}, g\right), m \geq 1$. Then $g_{m}$ is u.s.c. and $g_{m} \downarrow g$. Dini's theorem now applies to yield that the convergence $g_{m} \downarrow g$ is uniform on $Z$. Hence

$$
\underset{m}{\limsup } f_{m}\left(g_{m}\right) \leq \lim g_{m}\left(z_{m}\right)=g\left(z_{0}\right) \leq f\left(z_{0}\right)+\varepsilon .
$$

Proof of Theorem A. By [5, Theorem 5], for each $n \geq 1$, there is a $\mathscr{T}$ measurable selector $g_{n}: T \rightarrow Y$ of $G$ such that

$$
v_{n}\left(t, g_{n}(t)\right)=\sup _{y \in G(t)} v_{n}(t, y)
$$

for every $t \in T$.

For $t \in T$ set $H(t)=\left\{y \in G(t)\right.$ : there is a subsequence $\left\{g_{n i}(t)\right\}$ such that $\left.g_{n i}(t) \rightarrow y\right\}$. Plainly, $H(t)$ is nonempty, compact as $G(t)$ is. We claim that $H$ is $\mathscr{T}$-measurable. Let $C$ be closed in $Y$. Then

$$
\{t \in T: H(t) \cap C \neq \varnothing\}=\bigcap_{k \geq 1} \bigcup_{m>k}\left\{t \in T: \rho\left(g_{m}(t), C\right)<\frac{1}{k}\right\},
$$

where $\rho$ is a metric on $Y$. Since the set on the right belongs to $\mathscr{T}, H$ is $\mathscr{T}$-measurable. Use the Kuratowski-Ryll-Nardzewski selection theorem [3] to get a $\mathscr{T}$-measurable selector $g: T \rightarrow Y$ of $H$.

To complete the proof, fix $t \in T$ and let $y^{*}=g(t)$. So there is a subsequence $\left\{g_{n_{i}}(t)\right\}$ such that $y_{n_{i}}=g_{n_{i}}(t) \rightarrow y^{*}$. By condition (1) and Lemma B, we have

$$
\lim _{i} v_{n_{i}}\left(t, y_{n_{i}}\right) \leq v\left(t, y^{*}\right) .
$$

It follows that $v^{*}(t)=v(t, g(t))$.

\section{REFERENCES}

1. L. E. Dubins and L. J. Savage, Inequalities for stochastic Processes, Dover, New York, 1976.

2. C. J. Himmelberg, T. Parthasarathy, and F. S. Van Vleck, Optimal plans for dynamic programming, Math. Oper. Res. 1 (1976), 390-394.

3. K. Kuratowski and A. Mostowaski, Set theory, 2nd rev. ed., North-Holland, Amsterdam, 1976.

4. A. Maitra, Discounted dynamic programming on compact metric spaces, Sankhyā A $\mathbf{3 0}$ (1968), 211-216.

5. A. Maitra and B. V. Rao, Generalizations of Castaing's theorem on selectors, Colloq. Math. 44 (1981), 99-104. 
6. A. S. Nowak and T. E. S. Raghavan, Existence of stationary correlated equilibria with symmetric information for discounted stochastic games, preprint, 1989.

7. U. Rieder, Measurable selection theorems for optimization problems, Manuscripta Math. 24 (1978), 115-131.

8. M. Schäl, A selection theorem for optimization problems, Arch. Math. 25 (1974), 219-224.

9. __ Conditions for optimality in dynamic programming and for the limit of $n$-state optimal policies to be optimal, Z. Wahrsch. Verw. Gebiete 32 (1975), 179-196.

10. R. M. Solovay, A model of set theory in which every set of reals is Lebesgue measurable, Ann. of Math. (2) 92 (1970), 1-56.

11. D. J. Wagner, Survey of measurable selection theorems, SIAM J. Control Optim. 15 (1977), 859-903.

Department of Philosophy, Princeton University, Princeton, New Jersey 08544-1006

Department of Mathematics, Santa Fe Institute, Santa Fe, New Mexico 87501 